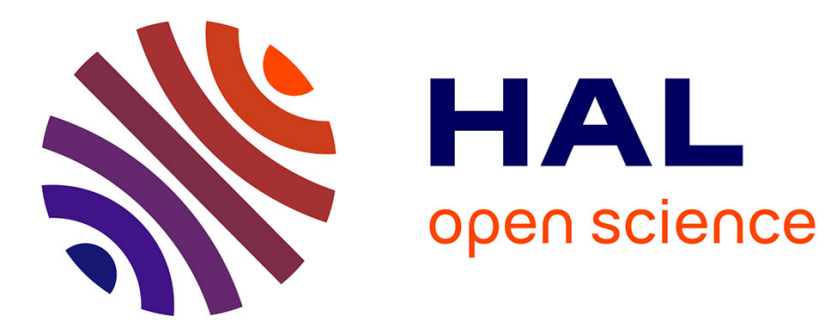

\title{
Heterodyne detection of multiply scattered monochromatic light with a multipixel detector
}

M. Gross, P. Goy, B. C. Forget, Michael Atlan, F. Ramaz, A. C. Boccara, A. K. Dunn

\section{- To cite this version:}

M. Gross, P. Goy, B. C. Forget, Michael Atlan, F. Ramaz, et al.. Heterodyne detection of multiply scattered monochromatic light with a multipixel detector. Optics Letters, 2005, 30, pp.1357-1359. 10.1364/OL.30.001357 . hal-00777798

\section{HAL Id: hal-00777798 https://hal.science/hal-00777798}

Submitted on 18 Jan 2013

HAL is a multi-disciplinary open access archive for the deposit and dissemination of scientific research documents, whether they are published or not. The documents may come from teaching and research institutions in France or abroad, or from public or private research centers.
L'archive ouverte pluridisciplinaire HAL, est destinée au dépôt et à la diffusion de documents scientifiques de niveau recherche, publiés ou non, émanant des établissements d'enseignement et de recherche français ou étrangers, des laboratoires publics ou privés. 


\title{
Heterodyne detection of multiply scattered monochromatic light with a multipixel detector
}

\author{
M. Gross, P. Goy \\ Laboratoire Kastler Brossel \\ Departement de Physique de l'Ecole Normale Superieure \\ 24, rue Lhomond F-75231 Paris cedex 05, France \\ B.C. Forget, M. Atlan, F. Ramaz, A.C. Boccara \\ Laboratoire d'Optique, Ecole Supérieure de Physique et de Chimie Industrielles de la Ville de Paris CNRS UPRA0005, \\ Université Pierre et Marie Curie, 10 rue Vauquelin 75231 Paris cedex 05
}

\author{
A.K. Dunn \\ Martinos Center for Biomedical Imaging, Massachusetts General Hospital, Charlestown MA
}

\begin{abstract}
We propose to study the broadening of the multiply scattered light due to the scatterers motion by a new technique which competes favorably with standard laser Doppler, and time varying speckle techniques. In our method, the scattered light is detected by a heterodyne receiver which uses a CCD as a multi pixel detector. The frequency spectrum of the scattered light is obtained by sweeping the heterodyne local oscillator frequency. Our detection scheme combines a high optical etendue (product of the surface by the detection solid angle) with an optimal detection of the scattered photons (shot noise). To demonstrate our technique, we have measured, in vivo, the frequency spectrum of the light scattered through $4 \mathrm{~cm}$ of human breast tissues.
\end{abstract}

\section{Citation}

M. Gross, P. Goy, B. C. Forget, M. Atlan, F. Ramaz, A. C. Boccara, and A. K. Dunn, "Heterodyne detection of multiply scattered monochromatic light with a multipixel detector," Opt. Lett. 30, 1357-1359 (2005) http://www.opticsinfobase.org/ol/abstract.cfm?URI=ol$30-11-1357$

We are involved in the development of acousto optical imaging techniques of thick biological tissues [1] (e.g. breast imaging), in which the temporal evolution of the speckle is an important limitation of the performances. To analyze the speckle, we have developed a technique, inspired from a previous work [2], able to perform an optimal, shot noise limited, heterodyne detection of the scattered light on a 2D detector. We have used our method to measure, in vivo, the frequency spectrum of the light scattered, in a transmission geometry, through the breast of a volunteer woman. We consider that our technique has a broader range of application in the domain of dynamic light scattering, and this letter intends to alert this community.

Coherent light (laser) scattering from moving objects or particles produces intensity fluctuations that can be used to measure the spectral (Doppler) broadening due to the motion of the scatterers. Two equivalent approaches [3], have been used to analyze these fluctuations: laser Doppler, and time varying speckle analysis. In laser Doppler (see introduction to feature issues in Applied Optics $[4,5])$, the scattered light is detected in the far field by a mono detector (photo diode or photo multiplier), and analyzed by a correlator or a spectrum analyzer. The sensitivity can be very high, in particular when the scattered light is mixed with a LO beam (local oscillator) [6]. However, the optical etendue (product of the surface by the detection solid angle) is low because the scattered light is collimated with pinholes, or with optical fibers [7]. In speckle contrast analysis techniques, the scattering object is imaged, without mixing (self-beating detection), on the detector, which can be advantageously 2D (photographic plate [8], CCD [9] or CMOS camera [10]). Accounting the space-time relationship of the correlation functions [11], one can get information on the scatterers motion [10, 12-14]. With 2D detection, speckle techniques benefit of a high etendue, but do not benefit on the LO beam mixing. Our technique combines the advantages of laser Doppler with LO beam mixing (high sensitivity) and speckle analysis (high etendue).

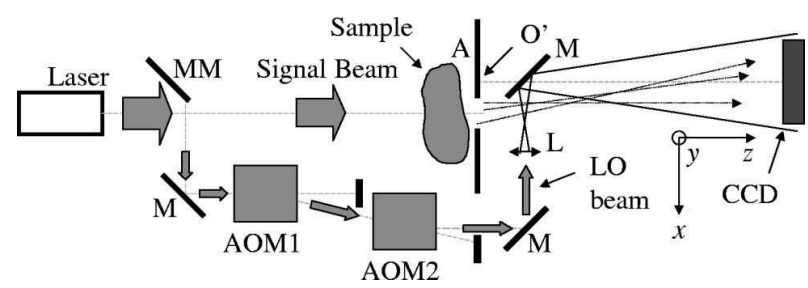

FIG. 1: a) Experimental setup. AOM1,AOM2: Acousto-optic modulators. MM: moving mirror. M: mirror. L: short focal lens. A: rectangular aperture. BS: beam splitter. CCD: CCD camera.

Fig 1 shows the experimental setup. The $780 \mathrm{~nm}$, $50 \mathrm{~mW}$ beam of the laser (Sanyo DL-7140-201, current: $95 \mathrm{~mA})$ is split in two beams. The main (>90\% of the power) beam (frequency $\nu_{L}$ ) is scattered by the sample (signal beam). The lower intensity beam $(<10 \%)$ is the LO of the CCD heterodyne detection. The respective power of the 2 beams is adjusted by moving 

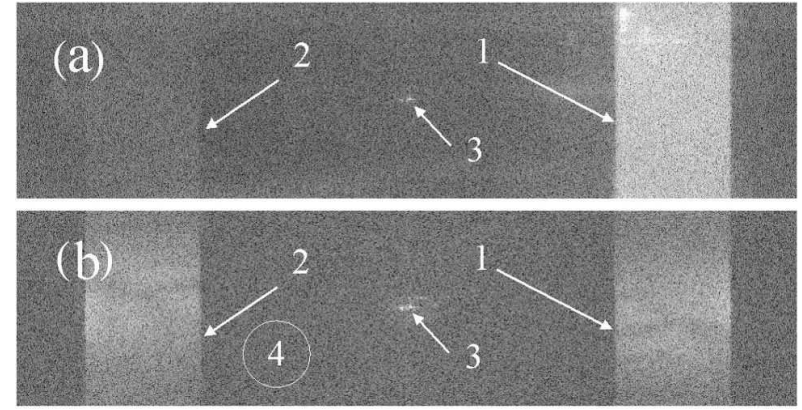

FIG. 2: Aperture reconstructed image $(1024 \times 256$, PCO camera). The main beam is diffused by a gel of latex spheres (a), and by $5 \mathrm{~mm}$ in vivo human tissues.

MM. By using the AOM1 and AOM2 acousto optic modulators (Crystal Technology: $\nu_{A O M 1,2} \simeq 80 \mathrm{MHz}$ ), the LO beam frequency $\nu_{L O}$ can be freely adjusted: $\nu_{L O}=\nu_{L}+\left(\nu_{A O M 1}-\nu_{A O M 2}\right)$. Two numerical synthesizers with the same $50 \mathrm{MHz}$ quartz reference clock provide the $\nu_{A O M 1,2} \mathrm{RF}$ signals. The lens $\mathrm{L}(25 \mathrm{~mm})$ focuses the LO beam in an equivalent point $\mathrm{O}$ ' located in the plane of the A vertical slit $(5.5 \mathrm{~mm})$ located 1 to $3 \mathrm{~cm}$ from the sample. The $\mathrm{LO}+$ signal interference pattern is recorded by the CCD camera: PCO Pixelfly (digital $12 \mathrm{bits}, \nu_{C C D}=12.5 \mathrm{~Hz}$ exposure time $80 \mathrm{~ms}$ with $1280 \times 1024$ pixels of $6.7 \times 6.7 \mu \mathrm{m})$ with is located at typically $30 \mathrm{~cm}$ from the slit. The camera is used in $\times 4$ vertical binning mode $(50 \mathrm{~Hz}, 1280 \times 256)$.

Because of the LO beam spacial coherence, the CCD camera records digital holograms [15]. By choosing $\nu_{L O}=\nu_{L}+\nu_{C C D} / 4$, we have recorded sequences of 4 CCD images $\left(I_{0} \ldots I_{3}\right)$, and calculated the complex field $E$ (i.e. the complex hologram) by the 4 -phase heterodyne variant[16] of the phase-shifting method [17]: $E=\left(I_{0}-I_{2}\right)+j .\left(I_{1}-I_{3}\right)$. We have calculated the field $E^{\prime}$ in the plane of the aperture A, i.e. we have reconstructed the image of the aperture (which is back illuminated by the sample). Because O' is within the A plane, the calculation of $E^{\prime}$ is made with a simple Fourier transform: $E^{\prime}=F T_{x, y}(E)$.

Fig.2a shows in logarithmic scale the intensity image $\left(\left|E^{\prime}\right|^{2}\right)$ obtained with a highly diffusing scattering sample (10\% PVA in water, scattering coefficient $\mu_{s}=3.9 \mathrm{~cm}^{-1}$, thickness $3 \mathrm{~cm}$ ). The scatterers are not moving, and the scattered photons remains within $\nu=\nu_{L}$ frequency delta function. The heterodyne detection, whose bandwidth is narrow $\left(\approx \nu_{C C D}\right)$, is fully efficient. The image of the aperture (1) is bright. By making the heterodyne detection on the other sideband (with $\nu_{L O}=\nu_{L}-\nu_{C C D} / 4$ ), one gets a reversed image, with the aperture on the left hand side. The twin image [15] seen in (2) corresponds thus to the $\nu_{L O}-\nu_{C C D} / 4=\nu_{L}-\nu_{C C D} / 2$ signal. It is low because scattered are not moving $\left(\nu=\nu_{L}\right.$ delta function). The zero grating order (3) parasitic image corresponds to the the LO beam. It is quite low, because the

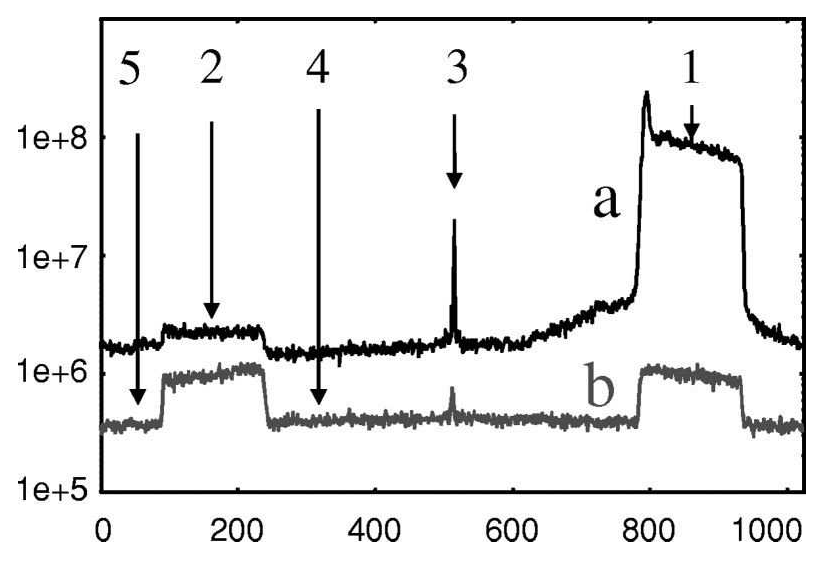

FIG. 3: Sum $S(x)$ over the $y$ axis of the images of Fig.2 for the gel sample (a), and the in vivo sample (b).

LO contributions cancel out in the 4-phase calculation of E.

Fig.2b is obtained with moving scatterers. The main beam travel through the medius-ring finger commissure ( $5 \mathrm{~mm}$ thick). The motion of the scatterers Doppler broadens the scattered light $(\approx 300 \mathrm{~Hz})$. The heterodyne detection is less efficient, and the brightness of the aperture image (1) is lower. Moreover, one get roughly the same signal on the two heterodyne sideband $\left(\nu_{L O} \pm \nu_{C C D} / 4\right)$, and the twin image (2) is as bright as the image itself (1). The zero grating order parasite (3) is still low.

To make a quantitative measurement of the scattered photons signal, we have plotted the sum $S(x)$ of the intensity signal over the $y$ axis: $S(x)=\int\left|E^{\prime}(x, y)\right|^{2} . d y$ (Fig.3). With the gel sample (a), the aperture rectangular peak (arrow 1) is much higher. With the tissue (b) the aperture peaks (arrows 1,2) are lower, and roughly symmetric. The zero order parasites (arrow 3) correspond to an excess noise of several of magnitude, but on a very localized region.

To explore the Doppler profile of the scattered photon, we have offset the frequency of the LO beam: $\nu_{L O}-$ $\nu_{L}=\Delta \nu=0,0.1,0.3,1 k H z(\mathrm{c}, \mathrm{d}$, e and $\mathrm{f})$. To get a better signal, we have recorded sequences of 2 images $\left(I_{0}, I_{1}\right)$, and calculated 2 phase holograms $\left(E=I_{0}-I_{1}\right)$. We have then averaged the intensity data $\left(\left|E^{\prime}\right|^{2}\right)$ over 64 sequences. As seen the scattered photon signal (arrows 1,2) decreases with the offset.

The signal observed in the regions masked by the aperture (arrows 4,5 ) is very flat. It is roughly the same as without signal (with LO beam alone). Accounting the PCO camera gain of $2.4 e$ (photo electron) per count (saturation means 4096 counts), it is, within $10 \%$, equal to LO beam shot noise. Since the shot noise corresponds, on the signal beam, to $1 e$ per pixel (or per field mode) within the measurement time, the noise floor provides us with an absolute calibration of the signal. As an example, on Fig. $4 \mathrm{c}$ ) the noise floor (arrows 4,5 ) is $1 e$, the signal 


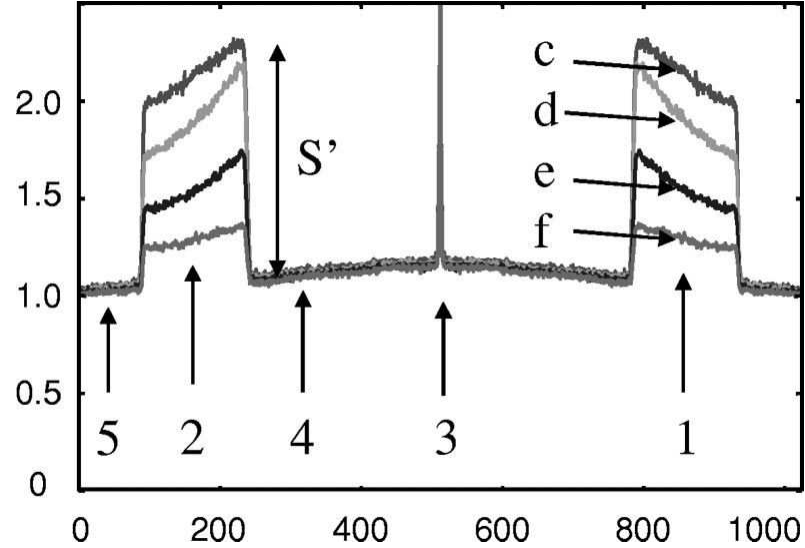

FIG. 4: Sum $S(x)$ for the in vivo sample with a LO frequency offset $\Delta \nu=0, .1, .3$ and $1 \mathrm{KHz}(\mathrm{c}, \mathrm{d}, \mathrm{e}$ and $\mathrm{f})$

(arrows 1,2 ) is $2 e$, and the signal, after noise correction and calibration, is $S^{\prime}=2-1=1 e$.

To illustrate the ability of our technique to get pertinent information with very low signal, we have recorded the frequency spectrum of a light beam that had travelled through the breast a a volunteer woman $(4 \mathrm{~cm}$ width). The breast is gently pressed between 2 transparent PMMA plates. A Hitachi KPF2B $(658 \times 496$ pixels of $7.4 \times 7.4 \mu \mathrm{m}$, quantum efficiency $25 \%$ at $780 \mathrm{~nm}$, exposure time $16 \mathrm{~ms}$ ) analog CCD camera is used. A Matrox Meteor frame grabber grabs the odd lines images $(640 \times 240$ pixels) continuously at $\nu_{C C D}=60 \mathrm{~Hz}$.

12800 images are grabbed in $213 s$, while $\Delta \nu$ is swept from 0 to $6 \mathrm{KHz}$ in 100 frequency intervals, each of them corresponding to 64 sequences of 2 images: $100 \times 64 \times$ $2=12800$. Half of the frequencies are over offset by $10 \mathrm{KHz}(\Delta \nu=10 \ldots 16 \mathrm{KHz})$. Since this offset is large with respect to the observed half-linewidth $(1.5 \mathrm{KHz})$, one get by this way a reference level without diffused photons.

For each sequence, we have made 2 phases detection, and we have calculated $S(x)$. For each frequency, we have averaged $S(x)$ over the 64 sequences: $\langle S(x)\rangle=$ $(1 / 32) \sum S(x)$. The signal + noise spectral component $P_{S+N}(\Delta \nu)$ of the scattered light is obtained then by summing $\langle S\rangle$ over the $x$ interval that corresponds to the aperture region 1: $\left.P_{S+N}=\int_{1} d x<S(x)\right\rangle$. The noise component $P_{N}$ is obtained by summing over an equal size interval that corresponds to a flat region like 4 or 5 .

The Fig. 5 shows the $\Delta \nu$ frequency spectrum of the light diffused by the breast. Curve a shows the normal- ized and corrected signal $P_{S}=\left(P_{S+N}-P_{N}\right) / P_{N}$, and curve b the zero signal reference. At maximum $(\Delta \nu=0)$, the signal is $\approx 0.05 \times$ the shot-noise (which is equal to 1 photo electron per CCD pixel during the measurement time in the measurement bandwidth [2]: $33 \mathrm{~ms}$ and $30 \mathrm{~Hz}$ for 2 phases measurement), and the signal to noise ratio is $\approx 5$. We observe a spectrum with a half-width of $\approx 1.5 \mathrm{KHz}$. This width is comparable with the one ob-

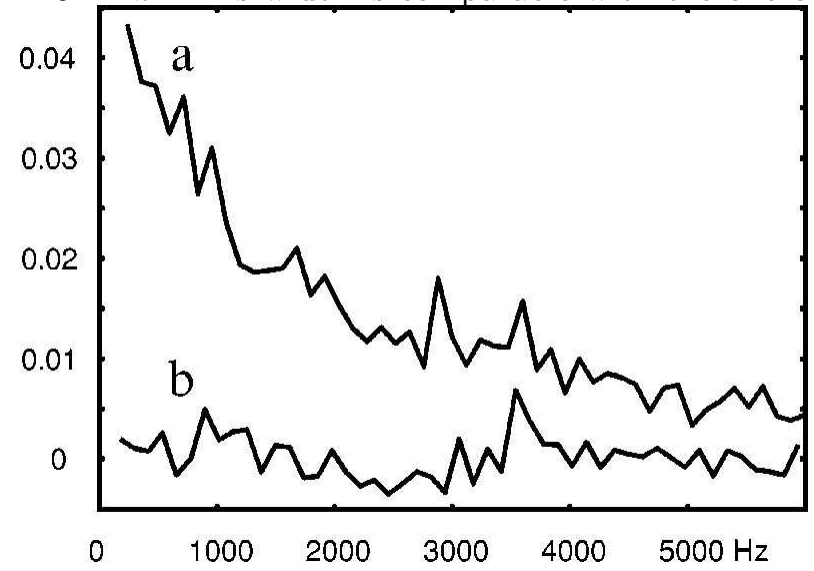

FIG. 5: a) Frequency spectrum of the light diffused by the breast $(\Delta \nu=0 \ldots 6 \mathrm{KHz})$. b) zero signal reference $(\Delta \nu=$ $10 \ldots 16 \mathrm{KHz})$.

tained by [18] on human wrist. This result is important for acousto optical imaging with CCD $[1,18,19]$, because the sensitivity of that technique decreases linearly with the Doppler broadening.

In conclusion, we have achieved a study of light scattered by thick living biological tissue with optimal (shot noise) sensitivity and with high etendue due to the parallel detection on a large number of detectors (pixels of a CCD camera). Our technique is frequency selective with a bandwidth fixed by the camera frame rate. The detection frequency is controlled by the AOM and can easily be scanned to obtain a the frequency profile of the scattered light.

An added advantage of using of our technique is that the alignment of our setup is very easy. With a multipixel detector there is no restrictive mode matching condition. The LO beam is within one or a few mode while the detection is made over the whole modes sustained by the multipixel detector. Here the multipixel detector allows us to perform a coherent detection of spatially incoherent light.
[1] S. Lévêque, A. C. Boccara, M. Lebec, and H. SaintJalmes, "Ultrasonic tagging of phton paths in scattering media: parallel speckle modulation processing," Opt. Lett. 24, 181 (1999).
[2] M. Gross, P. Goy, and M. Al-Koussa, "Shot-noise detection of ultrasound-tagged photons in ultrasoundmodulated optical imaging," Opt. Lett. 28, 2482-2484 (2003). 
[3] J. Briers, "Laser Doppler and time-varying speckle: a reconciliation," JOSA A 13, 345 (1996).

[4] W. V. Meyer, A. E. Smart, R. G. W. Brown, and M. A. Anisimov, "Photon correlation and scattering: introduction to the feature issue," Applied Optics 36, 7477 (1997).

[5] W. V. Meyer, A. E. Smart, and R. G. W. Brown, "Photon correlation and scattering: introduction to the feature issue," Applied Optics 40, 3965 (2001).

[6] R. Brown, "Homodyne optical fiber dynamic light scattering," Applied Optics 40, 4004 (2001).

[7] R. Brown, "Dynamic light scattering using monomode optical fibers," Applied Optics 26, 4846 (1987).

[8] A. Fercher and J. Briers, "Flow visualization by means of single-exposure speckle photography," Optics Com. 37, 326 (1981).

[9] J. Briers and S. Webster, "Quasi real-time digital version of single-exposure speckle photography for full-field monitoring of velocity or flow fields," Optics Com. 116, 36 (1995).

[10] A.Serov, W. Steenbergen, and F. de Mul, "Laser Doppler perfusion imaging with complementary metal oxide semiconductor image sensor," Optics Letters 27, 300 (2002).

[11] T. Yoshimura, "Statistical properties of dynamic speckles," JOSA. A 3, 1032 (1986).
[12] N. Takai, T.Iwai, T.Ushizaka, and T.Asakura, "Velocity measurement of the diffuse object based on timedifferentiated intensity fluctuations," Optics Com. 30, 287 (1979).

[13] A. F. Fercher, "Velocity measurement by first order statistics of the of time-differentiated laser speckles," Optics Com. 33, 129 (1980).

[14] P. Starukhin, S. Ulyanov, E. Galanzha, and V. Tuchin, "Blood-flow measurements with small number of scattering events," Applied Optics 39, 2823 (2000).

[15] U. Schnars and W. Jüptner, "Direct recording of holograms by a CCD target and numerical reconstruction," Appl. Opt. 33, 179-181 (1994).

[16] F. LeClerc, L. Collot, and M. Gross, "Numerical Heterodyne Holography Using 2D Photo-Detector Arrays," Optics Letters 25, 716 (2000).

[17] I. Yamaguchi and T. Zhang, "Phase-Shifting digital holography," Optics Letters 18, 31 (1997).

[18] A. Lev and B. Sfez, "In vivo demonstration of the ultrasound-modulated light technique," J. Opt. Soc. Am. A 20, 2347 (2003).

[19] L. Wang, S. Jacques, and X. Zhao, "Continuous wave ultrasonic modulation of scattered light to image objects in turbid media," Optics Letters 20, 629 (1995). 